\title{
¿El crecimiento de la matrícula en educación superior en Risaralda está produciendo una "generación engañada"?
}

\author{
Patricia Carvajal Olaya ${ }^{1}{ }^{(\mathbb{D}}$, René Guevara Ramírez \\ Universidad Tecnologica de Pereira, Universidad Pedagógica Nacional, Colombia
}

Autor de correspondencia:

1.pacarva@utp.edu.co

Recibido: 20 de septiembre de 2020

Revisado: 25 octubre de 2020

Aprobado: 18 de mayo de 2021

Publicado: 23 de junio de 2021

\section{Resumen}

A través de la investigación documental y de cifras estadísticas, el presente artículo recoge los hechos históricos, económicos, sociales y culturales alrededor de la configuración y el desarrollo de la educación superior en el departamento de Risaralda, en un entorno económico que, desde hace algún tiempo, no logra la dinámica de crecimiento necesaria para propiciar un medio laboral, en el que las presentes y futuras generaciones se puedan desarrollar profesionalmente, ya que se ha venido profundizando una brecha entre el número de graduados y las plazas ofertadas por el mercado laboral. Mediante datos objetivos, se pretende mostrar que la sobreabundancia de titulaciones en un mercado laboral contraído dificulta que los nuevos profesionales cumplan sus expectativas de llegar a mejores espacios laborales. Así, surge la inquietud sobre si en Risaralda se está presentando lo teorizado por Bourdieu acerca de la construcción de una "generación engañada", que apostó por la educación para adquirir conocimiento, que tiene derechos otorgados en los títulos y que necesita - mas no logra alcanzar- posiciones de mayor reconocimiento dentro del espacio social.

Palabras clave: desempleo, dinámica económica, educación superior, título universitario 


\title{
Is the growth of higher education enrollment in Risaralda leading to a "cheated generation"?
}

\begin{abstract}
Through documentary research and statistical data, this article compiles historical, economic, social, and cultural facts regarding the configuration and development of higher education in the department of Risaralda, in an economic environment that, for some time now, has not achieved the necessary dynamics of growth to provide a working environment in which present and future generations can develop professionally, since the gap between the number of graduates and the vacancies offered by the employment market has been widening. Using objective data, the aim is to prove that the overabundance of graduates in a shrinking employment market makes it difficult for new professionals to fulfil their expectations of finding better job opportunities. Consequently, a question emerges about whether what Bourdieu theorized on the creation of a "cheated generation" is taking place in Risaralda, a generation that bet on education to acquire knowledge, that has rights granted in the titles, and that needs-but fails to achieve-positions of greater recognition within the social space.
\end{abstract}

Keywords: unemployment, economic dynamics, higher education, university degree

\section{O crescimento da inscrição no ensino superior em Risaralda está produzindo uma "geração enganada"?}

\section{Resumo}

Através da pesquisa documental e dados estatísticos, este artigo recolhe fatos históricos, econômicos, sociais e culturais a respeito da configuração e desenvolvimento do ensino superior no departamento de Risaralda, num ambiente econômico que, há algum tempo, não tem alcançado a dinâmica de crescimento necessária para propiciar um ambiente de trabalho no qual as gerações presentes e futuras possam se desenvolver profissionalmente, pois a disparidade entre o número de graduados e as vagas oferecidas pelo mercado de trabalho vem se ampliando. Através de dados objetivos, o propósito é provar que a superabundância de graduados num mercado de trabalho reduzido torna difícil para os novos profissionais satisfazer suas expectativas de encontrar melhores oportunidades de trabalho. Assim, surge uma questão sobre se o que Bourdieu teorizou sobre a construção de uma "geração enganada" está ocorrendo em Risaralda, geração que apostou na educação para adquirir conhecimento, que tem direitos concedidos nos títulos e que necessita - mas não consegue alcançar - posições de maior reconhecimento dentro do espaço social.

Palavras-chave: desemprego, dinâmica econômica, educação superior, diploma universitário 
${ }^{1}$ La Universidad Tecnológica de Pereira (UTP) se creó como respuesta a la necesidad de mayor cantidad de mano de obra calificada en el departamento de Risaralda. Esta necesidad se presentaba en otras regiones del país, en pleno auge del modelo propuesto por la Comisión Económica para América Latina y el Caribe $\left(\mathrm{Cepal}^{2}\right)$ de industrialización por sustitución de importaciones $\left(\mathrm{ISI}^{3}\right)$, cuya lógica se validó, ya que los países con mayores desarrollos y economías más fuertes, luego de la Segunda Guerra Mundial, eran precisamente los que se habían industrializado, es decir, aquellos que fabricaban sus propios bienes de consumo interno e incluso estaban en capacidad de elaborar productos para exportar. Desde este panorama, fundar una institución de educación superior (IES), en un departamento también naciente, brindaba la oportunidad de participar en el triángulo de desarrollo estado-empresa-universidad, en aras de contribuir al progreso económico de la región y, por ende, del país, a través de la introducción de ciencia, tecnología e innovación para fortalecer la industria.

Sin embargo, como se mostrará en los siguientes apartados, la demanda interna en el departamento fue insuficiente y limitó la vinculación con la ciencia y la tecnología. La red industrial era poco densa y escasamente diversificada. El empresariado industrial tenía experiencia específica principalmente en agroexportación, dedicado ante todo al sector primario ${ }^{4}$. En este contexto, puede deducirse que no se logró el impulso suficiente para promover la actividad industrial (Hurtado, 2018). Sin el crecimiento de los sectores productivos, se fue generando paulatinamente un estancamiento de la economía, con las correspondientes consecuencias como la caída de la actividad productiva y, por lo tanto, una semiparálisis del mercado laboral en la región. Aun así, la UTP continuó otorgando sistemáticamente títulos profesionales a los jóvenes de la región, quienes en su gran mayoría han sido de origen social humilde. Tanto ellos como sus familias han apostado por la educación como alternativa para lograr movilidad social ascendente, expectativa incumplida dadas las condiciones antes descritas.

En el primer apartado, se presentará el contexto histórico y sociocultural del desarrollo del departamento de Risaralda con énfasis en la manera como se fueron configurando y concretando la idea de impulsar la educación en todos los niveles educativos y especialmente el propósito de ofertar educación superior pública para los habitantes propios y de regiones aledañas que se caracterizan por tener escasos capitales.

En el segundo apartado, se menciona el contexto económico y laboral del departamento al que se enfrentan los egresados de la educación superior, encuadrado en el modelo de ISI. Desde 1977, ya se preveía una dinámica lenta de desarrollo económico, evidenciada en

\footnotetext{
1 El presente artículo es parte del tercer capítulo de tesis doctoral en Educación, titulada Los persistentes de la UTP. ¿En qué condiciones sociales algunos estudiantes universitarios clasificados en alto riesgo de deserción continúan sus estudios? En esta se aplican complementariamente la investigación cualitativa y cuantitativa.

2 "Es una de las cinco comisiones regionales de las Naciones Unidas fundada en 1948 para contribuir al desarrollo económico de América Latina, coordinar las acciones encaminadas a su promoción y reforzar las relaciones económicas de los países entre sí y con las demás naciones del mundo". (Cepal, 2021).

3 El modelo de ISI es un aporte significativo de la teoría desarrollada por la Cepal durante los años 40, que, frente al estancamiento de las economías de la región, propone el desarrollo del sector industrial, en especial de las pequeñas y medianas empresas, encargadas de la producción de los insumos para consumo en cada país y, en consecuencia, del aumento del empleo y de la disminución de la dependencia de mercados extranjeros (Escobar, 2018).

4 El sector primario es el encargado de extraer directamente los bienes que proporciona la naturaleza, sin modificarlos en un proceso industrial: básicamente agricultura y ganadería.
} 
bajas tasas de ocupación, altas tasas de desempleo y el poco aporte del departamento al PIB nacional.

En el último apartado, se analiza lo denominado el escalamiento de la "generación engañada" (Bourdieu, 1979). Se muestra cómo la expansión de la educación superior y el otorgamiento de títulos en una región en la que el mercado laboral está contraído genera un desequilibrio entre las esperanzas subjetivas y las posibilidades objetivas de los jóvenes profesionales (Bourdieu \& Wacquant, 2005), en especial de aquellos que, por su proveniencia social, no disponen de un acumulado de capitales que les permita competir por los escasos puestos laborales mejor posicionados. Es decir, la desigualdad de la estructura social se reproduce y los individuos provenientes de sectores ventajosos mantienen su posición privilegiada, mientras que los de otros sectores sociales tienen pocas probabilidades de movilizarse dentro del espacio social, a pesar de sus altos niveles de escolaridad, especialmente, cuando hay sobreabundancia de títulos en el mercado.

La investigación es cualitativa y se basa en una revisión documental y una compilación de cifras estadísticas de los hechos históricos, económicos, sociales y culturales ocurridos en el desarrollo de Risaralda desde 1938 hasta 2018, con énfasis en el crecimiento de la educación superior en el departamento, visto a través del — cada vez mayor— número de graduados.

\section{Contexto histórico y sociocultural del inicio de la educación superior en Risaralda}

Risaralda es uno de los 33 departamentos que conforman la nación colombiana. Posee un territorio de $3585 \mathrm{~km}^{2}$ y está ubicado en la región andina, en el centro occidente del país. Fue fundado en 1966. Su capital es Pereira y, junto con otros 13 municipios que conforman el departamento, ha crecido poblacionalmente según muestra la tabla 1.

Tabla 1

Crecimiento de la población de Risaralda

\begin{tabular}{cccccccccc}
\hline Año & $\mathbf{1 9 3 8}$ & $\mathbf{1 9 5 1}$ & $\mathbf{1 9 6 4}$ & $\mathbf{1 9 7 3}$ & $\mathbf{1 9 8 0}$ & $\mathbf{1 9 8 5}$ & $\mathbf{1 9 9 5}$ & $\mathbf{2 0 0 5}$ & $\mathbf{2 0 1 8}$ \\
\hline \# de habitantes & 60492 & 309030 & 436759 & 498609 & 498609 & 710821 & 871588 & 897509 & 951943 \\
\hline
\end{tabular}

Nota. Los datos antes de 1967 representan a la ciudad de Pereira. Tomado de DANE, 1938,1951, $1964,1973,1985,2019$.

A lo largo de la historia, el desarrollo social, cultural y político de sus habitantes se ha sostenido económicamente en actividades como la agricultura ${ }^{5}$, la ganadería, la industria ${ }^{6}$ y -desde hace algún tiempo- el comercio. Dicho desarrollo fue liderado por las familias colonizadoras del territorio, que a su vez se apropiaron de las tierras aptas para el cultivo y la ganadería. Estas acumularon un importante capital económico que luego usaron para la creación de empresas dedicadas a la producción de bienes y servicios. El capital económico también permitió acumular capital político y, por ende, el poder. Prueba de ello es que en 1936 el Concejo Municipal estaba integrado por un total de 26 personas, todos reconocidos

\footnotetext{
5 Producción de café, caña de azúcar, plátano, yuca, cacao, piña, guayaba, papa, maíz, algodón y algunos frutales. En especial, el café soportó la economía del departamento de Risaralda. En 1905 el café de la zona representaba el $40 \%$ de la exportación del país y llegó a alcanzar el $70 \%$.

6 Alimentos, las bebidas, los textiles, el papel, el carbón y el vidrio.
} 
empresarios regionales ${ }^{7}$ (Zuluaga, 2013).

Como ha ocurrido históricamente en los espacios sociales, quienes acumulan grandes volúmenes de capital atesoran poder y se legitiman para trazar las líneas de desarrollo. De esta manera se consolidó una ciudad física, social y cultural, acorde con los criterios de la clase dirigente de la época, orientada a fomentar el progreso y las buenas costumbres. Prueba de ello fueron las intervenciones para definir qué y cómo se debería publicar en las radiodifusoras, en los medios escritos, en el control de la movilidad vehicular, en la regulación de avisos y carteles, en el diseño de las casas, entre otras (Acevedo et al., 2009).

En este propósito, los discursos entusiastas de civismo y de patriotismo fueron instrumentos movilizadores para lograr la participación de la comunidad en el apoyo a las ideas de progreso colectivo. También fue importante la reproducción de estos por parte de instituciones como familia, escuela e Iglesia, hasta conformar un habitus colectivo alrededor del progreso material -industria y comercio- y espiritual - artes, cultivo de las ciencias, fomento a la educación pública y ejercicio de las profesiones-. Lo anterior concuerda con Bourdieu (2002): "existe casi siempre, en toda sociedad, una pluralidad de potencias que en virtud de su poder político o económico están en condiciones de imponer sus normas culturales" (p. 31).

No obstante, el desarrollo industrial posterior a la crisis mundial de 1930 evidenció la necesidad de obreros cualificados en nuevas tecnologías e ingenieros especializados, que hicieran frente al nuevo mundo productivo tecnologizado. En respuesta a estas necesidades, la Sociedad de Mejoras Públicas de Pereira (SMPP) se movilizó en el fomento a la educación y la cultura. Gestionó recursos tanto para la infraestructura de colegios como de espacios de cultura en todas sus expresiones. Se resalta el agenciamiento de la construcción de instituciones escolares, de enseñanza secundaria, formación técnica e inclusive universitaria (Correa, 2014).

En ese momento, había dos grupos principales que definían el mapa social de Risaralda. En forma de oposición, había uno muy poderoso en virtud del volumen importante de capitales acumulados - reconocido como la "élite" de los dominantes- y el resto de los pobladores - o la "masa", los dominados-. El primero se enfocaba en mantener sus posiciones de poder a nivel local, a la vez que escalaba lugares en el Gobierno nacional, al utilizar como estrategia reproductiva la implantación de un discurso de civismo y patriotismo en el grupo conformado por habitantes externos a la élite. El ejercicio se facilitó gracias a las relaciones entre el grupo dominante y la institucionalidad: la Iglesia, los medios de comunicación y la escuela. Esto legitimó el estado de las cosas.

En especial, la escuela jugó un papel importante en la reproducción del discurso de civismo y con ello consiguió el apoyo irrestricto de la clase dirigente para ampliación de cobertura y mejoramiento de la infraestructura educativa, en armonía con las políticas nacionales en esta materia. Desde el Congreso se reglamentó que el $50 \%$ de impuesto predial recolectado por los municipios fuese orientado al fomento a la educación. Con ello

7 Cinco concejales dueños de la Cía. de Exportaciones; un concejal dueño de la Compañía Gutiérrez Mejía y Cía.; tres concejales dueños de la compañía Castro e Hijos; un concejal de Buitrago y Cía.; un concejal de Cano Botero y Cía., empresa de confecciones; un concejal hacendado; un concejal de la empresa Cano e Hijos; tres concejales de la compañía Echeverri y Cía.; tres concejales de la Cía. Exportadora del Pacífico; un concejal de la Cía. Industrial Campoalegre; un concejal de la Cía. Vidriera de Caldas; un concejal de la Cía. de Tranvías de Pereira; tres concejales de la Industria Harinera de Caldas; un concejal de Gaviria Jaramillo y Cía. 
se fortaleció la infraestructura física de los planteles educativos y se robusteció la planta docente. Otra iniciativa importante de impulso a la educación se incluyó en la Constitución del 91, en la que se reguló que "la educación [fuese] un derecho y un servicio público con función social para el rango de edad entre 5 y 15 años como responsabilidad del Estado". (art. 67).

En ese marco de lineamientos y de estrategias tomadas desde el ámbito político nacional, Risaralda incrementó la cobertura educativa. En primaria, creció un $15 \%$ entre 1973 y 2018; pasaron de 78160 a 90819 estudiantes. En secundaria, crecieron un 888 \% en el mismo periodo, al pasar de 9823 a 97020 estudiantes.

Como se mencionó, la educación superior llegaría a Risaralda a través de la SMPP, liderada en 1942 por el doctor Jorge Roa Martínez ${ }^{8}$, considerado en la historia de la región como el agente social que dedicó mayor capacidad de gestión para impulsar la configuración inicial de la educación superior en el departamento. En principio, apuntó a la consecución de recursos para construcción y dotación de un plantel orientado a formación agrícola y técnica; y luego, a la construcción de la UTP, que inició labores el 4 de marzo de 1961.

Según Acevedo et al. (2009), "Roa Martínez tuvo el poder de concretar muchas de las ideas que promovió, y que según Pierre Bourdieu es el rol propio del intelectual” (p. 27). Esta interpretación sobre Bourdieu puede leerse como que los intelectuales cumplen su función cuando concretan sus ideas y eso es precisamente lo que Roa logró. Fue un agente social con todas las posibilidades reales de acción y decisión, que llegó a Pereira en un momento histórico, con sus acumulados de capital cultural y político, y además con un ideario cívico y humanista que reforzó con amplio capital social al vincularse a la élite pereirana a través de una sociedad conyugal favorable, lo que le llevó a ocupar posiciones privilegiadas en la región, desde donde, por fortuna, gestionó en favor de las clases menos favorecidas, principalmente, a través del fomento de la educación superior.

Sostenido en una "fe ciega en la educación tecnológica para salvar al país de casi todos los males de la sociedad" (Acevedo et al., 2009, p. 272), mediante un liderazgo político y estratégico ejecutado frente a la SMPP9 conocida como "Sociedad de ideas", logró, en conjunto con los demás protagonistas de la toma de decisiones de ciudad, que se expidiera la Ley 41 del 15 de diciembre de 1958, con la cual el Congreso de la República funda la UTP, como universidad pública, para "la formación de ingenieros profesionales en las distintas ramas técnicas... necesarias para el desarrollo del país", con garantía del presupuesto para su funcionamiento y operación.

Obsérvese que se priorizó el orden jurídico y presupuestal frente a la planeación académica. Así, Roa, después de la aprobación para el funcionamiento de la UTP, se dedicó

\footnotetext{
8 Abogado, egresado de la Universidad Nacional de Colombia, gobernador de Boyacá, alcalde de Pereira, gerente del Banco Central Hipotecario. Fue miembro especial de la SMPP. Como intelectual reconocido, lideró importantes obras de desarrollo en la región, especialmente las relacionadas con infraestructura para la educación técnica y superior.

9 "El 2 de mayo de 1925, se dio inicio con la firma de la primera acta en el Club Colombia, a la Sociedad de Mejoras Públicas de Pereira, fundada por los hombres más distinguidos de la época. Su propósito es: 1) Promover una ciudadanía responsable y aportar en el cambio de mentalidad y cultura de ciudad. 2) Fomentar el espacio público de calidad, el buen uso del tiempo libre y la responsabilidad ambiental. 3)Articular al sector empresarial, la academia, el Estado, tercer sector y la comunidad organizada para aportar al desarrollo regional de largo plazo" (Sociedad de Mejoras Públicas de Pereira, 2021), entre sus logros están: la Cámara de Comercio, el aeropuerto Matecaña, traslado del Batallón San Mateo, Hospital San Jorge, estadio Olímpico, Universidad Tecnológica de Pereira, entre otras obras.
} 
a buscar información estadística sobre qué programas académicos se ofrecerían. Se registró su gestión ante instituciones públicas y privadas - ministerios, Dirección Nacional de Estadística, Asociación Nacional de Industrias, agremiaciones y demás- en busca de datos tanto de egresados de universidades del país como del exterior que estuviesen trabajando en fábricas. Este hecho evidencia el intento de Roa de planificar la oferta académica de acuerdo con necesidades de la región. Sin embargo, no consiguió la información deseada, ya que no había censos en el país que recogiesen ese tipo de datos (Acevedo et al., 2009).

Esta anotación histórica es una evidencia de que el otorgamiento de licencias de funcionamiento a IES y el crecimiento de estas no obedecieron a planes estructurados de desarrollo de una educación superior de alta calidad, lo que de alguna manera ya había señalado Rama (1970). Si bien el gobierno de Rojas Pinilla creó el Fondo Universitario Nacional (FUN) para: "proveer el desarrollo de las universidades colombianas" (Decreto 3686, 1954, art.1),, al parecer las actividades de la entidad en esta materia no fueron suficientes. Por tal razón, el 22 de junio de 1958 se fundó la Asociación Colombiana de Universidades. El trabajo conjunto para formular y controlar las políticas universitarias se divide nuevamente y el FUN deviene en el ICFES. Estas intenciones burocráticas para administrar la educación superior al parecer no han logrado su propósito, quizás porque "la expansión universitaria no se había constituido como el resultado de un plan" (Rama, 1970, p. 50).

\section{Contexto económico y laboral de la educación superior en Risaralda}

Este apartado tiene como finalidad mostrar el panorama laboral del departamento, de la región y del país, al cual se enfrentaban las primeras cohortes de profesionales egresados de la UTP. Se calcularon las cifras de desempleo nacional y regional con estos datos. Los resultados fueron $9,42 \%$ y $10,17 \%$, respectivamente.

Sin duda, en ese momento la apuesta por la educación tecnológica había permeado toda Latinoamérica. Se le consideraba una manera de salir del atraso económico de los países del continente. La tendencia desarrollista de los años 60, que viene desde los años 40, tras la idea de la Cepal de implementar el modelo de ISI, implicó la necesidad de formar mano de obra cualificada para impulsar el desarrollo, a través de la producción industrial propia de insumos y de bienes de capital ajustados a los requerimientos del país. Esto orientó el esfuerzo a la formación de un profesional de alta escuela en ingenierías (Acevedo et al., 2009).

En ese contexto, se iniciaron labores académicas en marzo de 1961 con 15 nuevos estudiantes universitarios, cifra que llegó a 65 matriculados en el primer año, 195 -en 1962-, 266 en 1963 - y 374 - en 1964 - valga decir, sin el requerimiento de un examen de admisión académico (Acevedo et al., 2009, p. 279). Además, de acuerdo con el registro histórico, desde el punto de vista económico, fueron estudiantes provenientes en su mayoría de estratos bajos. Según la clasificación revelada por la Secretaría Académica de la UTP (2011), el 72 \% de ellos tenía recursos "escasos"; el $22 \%$, "medios"; y el $6 \%$, "abundantes". Lo anterior coincide con el propósito de Roa, en cuanto a lograr una IES abierta a las "clases populares".

Es importante anotar que, mientras se abría la primera IES en Pereira, la ciudad ya no era la aldea de comienzos del siglo XX. Había alcanzado un crecimiento notorio en todos 
sus ámbitos, como se ve a continuación:

Comercial, existían 2100 negocios de comercio $^{10}$, once casas bancarias, cámara de comercio; industrial, un inventario de fábricas de producción de insumos, entre las que se contaban: una de cerveza, cuatro de gaseosas, diez de jabón y velas, dos de muebles metálicos, una de betún, tres de textiles, quince de café molido, una de medias, dos de vestidos para hombres, una de paños, una de confites y galletas; una de vidrios, una de papeles y algodón, metalmecánicas y de transformadores; una de ron; cultural, seis teatros o salas de cine, cuatro emisoras, dos diarios, seis semanarios, dos revistas; educación: tres escuelas de comercio, diecinueve escuelas urbanas y treinta rurales, ocho colegios de bachillerato para hombres y siete para señoritas; infraestructura: cinco parques, tranvía eléctrico, planta de luz eléctrica, red de acueducto y alcantarillado, planta telefónica (primera en Suramérica), plaza de ferias, plaza de mercado cubierta; salud: dos hospitales, dos clínicas oficiales y tres particulares; económico: pasó de un presupuesto de \$15 000 (1913) a \$16 500000 (1955). Movilidad: 3110 vehículos, 101 motocicletas, 3260 bicicletas. (Acevedo et al., 2009, p. 243)

El crecimiento y el desarrollo alcanzados por la ciudad se originaron en los esfuerzos propios de sus habitantes y muy poco debió agradecer a los apoyos del gobierno de Caldas, razón por la cual la dirigencia pereirana se enfocó en la búsqueda de independencia y autonomía presupuestal. Lo logró mediante la Ley 70 de 1966 del Congreso de la República, vigente desde el 1 de febrero de 1967, en la que se declaró a Pereira y a los otros 13 municipios como el nuevo departamento de Risaralda, con un total de 483461 habitantes y un presupuesto de \$66 982005 (Asamblea Departamental de Risaralda, 1968, Ordenanza 1).

De manera paralela a la creación del nuevo departamento de Risaralda, se graduaron los primeros estudiantes de la UTP, que entre 1966 y 1975 fueron en total 1308, el $95 \%$ de ellos como ingenieros y tecnólogos.

Los primeros graduados encontraron un entorno laboral regional cuyos indicadores económicos en términos de desempleo estaban por encima del indicador nacional. Así mismo, la tasa de ocupación estaba por debajo (tabla 2).

Tabla 2

Tasa de desempleo y tasa de ocupación nacional y Risaralda

\begin{tabular}{ccccc}
\hline & \multicolumn{2}{c}{ Nacional } & \multicolumn{2}{c}{ Risaralda } \\
\hline Año & 1975 & 1977 & 1975 & 1977 \\
\hline Tasa de desempleo & 10,6 & 9,8 & 16,8 & ND \\
Tasa de ocupación & 89,4 & 90,2 & 83,1 & ND \\
\hline
\end{tabular}

Nota.Tasa de desempleo = desocupados / PEA (población económicamente activa). Tasa de ocupación = ocupados / PET (población en edad de trabajar, considerada como mayor a 12 años en la zona urbana y mayor a 10 años en la zona rural). Los datos de Risaralda incluyen solo los datos de Pereira. Adapatada de Boletín mensual de estádistica del DANE, 1975 y 1977.

10 Pereira se ha conocido históricamente por su vocación comercial, a causa de dos razones principales. La primera es su ubicación geográfica, pues - como ciudad intermedia- está en el centro de lo que se conoce como "el triángulo de oro", formado por Bogotá, Medellín y Cali. Por lo tanto, es de paso obligado entre los caminos que comunican las tres capitales más grandes del país. La segunda es la inmigración de palestinos, sirios y libaneses a finales del siglo XIX, que radicaron sus comercios en la ciudad (Zuluaga, 2013, p. 484) 
En 1973 se llevó a cabo el XIV Censo Nacional de Población y III de Vivienda en Colombia (DANE, 1973). Las cifras revelaron que el país ascendió a 22862118 habitantes, con una PEA $^{11}$ de 6239 984, es decir, el equivalente al $27 \%$ del total, distribuida por nivel educativo como se muestra en la tabla 3. Además, el grueso de la fuerza laboral se encontraba en los primeros niveles de escolaridad: el $59.7 \%$ con primaria, un $16 \%$ con bachillerato y solo $2.7 \%$ con educación superior.

Estas cifras fueron motivo de preocupación para los entes gubernamentales, particularmente por el enfoque economicista con el que se concibió la educación y que, de alguna manera, se consignó en el Boletín mensual de estadística (1977): "el nivel educacional de la PEA tiene una función primordial en el desarrollo económico del país ya que la mayor o menor productividad de los factores de producción está asociada al nivel de capacitación de los recursos humanos" (p. 10).

La representación de la educación superior dentro de la fuerza de trabajo en 1973, a nivel nacional y regional, significó una oportunidad para los nuevos profesionales egresados de la UTP, en el marco de los avances de desarrollo tecnológico e industrial.

Tabla 3

PEA según nivel de escolaridad: nacional y Risaralda

\begin{tabular}{ccccc}
\hline \multicolumn{4}{c}{ Nivel de escolaridad de la PEA nacional y Región Central } & \multicolumn{3}{c}{ Censo de 1973- } \\
\cline { 1 - 4 } & Nacional & $\%$ & Región Central & $\%$ \\
\hline Primaria urbana & 2256013 & 36,2 & 678321 & 40,7 \\
Primaria rural & 1466596 & 23,5 & 370108 & 22,2 \\
Bachillerato básico & 261248 & 4,2 & 43753 & 2,6 \\
Bachillerato clásico & 727535 & 11,7 & 240747 & 14,4 \\
Sec. técnica o vocacional & 47961 & 0,8 & 4995 & 0,3 \\
Otra secundaria & 7500 & 0,1 & 3129 & 0,2 \\
Normal & 82638 & 1,3 & 18792 & 1,1 \\
Superior universitaria & 165724 & 2,7 & 27098 & 1,6 \\
Otra & 23435 & 0,4 & 3110 & 0,2 \\
Ninguna & 1201334 & 19,3 & 278026 & 16,7 \\
\hline Total & 6239984 & & 1668079 & \\
\hline
\end{tabular}

Nota. Región Central incluye: Risaralda, Caldas, Quindío, Tolima y Huila. Tomado de Boletín mensual de estadística del DANE, 1976, (297 y 298).

Dentro de la fuerza de trabajo, el grupo de los ocupados se distribuyó por posiciones ocupacionales según las ramas de actividad económica a las que podrían aspirar. En 1973, había un importante número de actividades de la economía sin especificar, así como trabajadores que no podían ser clasificados en ellas. Por lo anterior, y sin perder de vista los datos arrojados por el censo de 1973, se utilizaron los datos de la Encuesta de hogares de 1977, cuyo tamaño de muestra incluyó 2144198 habitantes, clasificados como ocupados dentro de la PEA. El dato fue acopiado por el DANE para las cuatro capitales más grandes del país y la distribución se puede ver en la tabla 4. Allí, los sectores de actividad

11 PEA: fuerza de trabajo, que puede estar ocupada o desocupada. 
económica con mayor número de trabajadores contratados fue servicios comunales sociales $y$ personales $^{12}-30,41 \%$-, seguido de la industria manufacturera $-25,72 \%$ - y luego el comercio, restaurantes $y$ hoteles $-20,54 \%$ -

Por otra parte, en términos de la distribución de trabajadores según posición ocupacional, se observa que el $64,3 \%$ son los empleados y obreros —estos últimos se consideran como personal manual asalariado, conocidos también como operarios-. El segundo grupo de ocupación con mayor contratación es el de trabajador por cuenta propia ${ }^{13}-21 \%$ -

\section{Tabla 4}

Ocupados por posición ocupacional según rama de actividad económica, 1977

\begin{tabular}{|c|c|c|c|c|c|c|c|c|}
\hline $\begin{array}{l}\text { Rama de la } \\
\text { actividad } \\
\text { económica }\end{array}$ & $\begin{array}{l}\text { Empleados } \\
\text { y obreros }\end{array}$ & $\begin{array}{c}\text { Servicio } \\
\text { doméstico }\end{array}$ & $\begin{array}{l}\text { Patrón o } \\
\text { empleador }\end{array}$ & $\begin{array}{c}\text { Trabajador } \\
\text { por cuenta } \\
\text { propia }\end{array}$ & $\begin{array}{c}\text { Trabajador } \\
\text { familiar sin } \\
\text { remuneración }\end{array}$ & $\begin{array}{c}\text { No } \\
\text { informa }\end{array}$ & $\begin{array}{l}\text { Total por } \\
\text { rama } \\
\text { económica }\end{array}$ & $\%$ \\
\hline Agropecuaria & 13385 & & 5633 & 5294 & 254 & & 24566 & 1,15 \\
\hline $\begin{array}{l}\text { Minas y } \\
\text { canteras }\end{array}$ & 3921 & & 392 & 0 & 0 & & 4313 & 0,20 \\
\hline $\begin{array}{l}\text { Industrias } \\
\text { manufactureras }\end{array}$ & 445917 & & 21920 & 77029 & 6562 & & 551428 & 25,72 \\
\hline $\begin{array}{l}\text { Electricidad, } \\
\text { gas y agua }\end{array}$ & 14369 & & 4182 & 0 & 0 & & 18551 & 0,87 \\
\hline Construcción & 93957 & & 28623 & 50824 & 818 & & 174222 & 8,13 \\
\hline $\begin{array}{l}\text { Comercio, } \\
\text { restaurantes y } \\
\text { hotel }\end{array}$ & 247507 & & 2984 & 167044 & 22928 & & 440463 & 20,54 \\
\hline $\begin{array}{l}\text { Transporte y } \\
\text { comunicación }\end{array}$ & 103675 & & 3732 & 26969 & 460 & & 134836 & 6,29 \\
\hline $\begin{array}{l}\text { Estable } \\
\text { financiero }\end{array}$ & 108532 & & 10350 & 24150 & 601 & & 143633 & 6,70 \\
\hline $\begin{array}{l}\text { Serv. comun. } \\
\text { soc. y pers. }\end{array}$ & 348078 & 201800 & & 98836 & 3341 & & 652055 & 30,41 \\
\hline No informa & 0 & & & & & 131 & 131 & 0,01 \\
\hline $\begin{array}{l}\text { Total por } \\
\text { posición } \\
\text { ocupacional }\end{array}$ & 1379341 & 201800 & 77816 & 450146 & 34964 & 131 & 2.144 .198 & \\
\hline$\%$ & 64,33 & 9,41 & 3,63 & 20,99 & 1,63 & 0,01 & & \\
\hline
\end{tabular}

Nota. Tomado de Boletín mensual de estadística del DANE, 1977.

Al profundizar en la composición de la contratación laboral en Colombia en 1977, según categorías ocupacionales, se observa en la tabla 5 que el grupo referente a profesionales $y$ técnicos solo representa el 9,8 \% del total. Precisamente, esa categoría laboral es la de mayor interés para los profesionales recién graduados de la UTP. En contraste, las categorías trabajadores y operarios no agrícolas y trabajadores de los servicios ${ }^{14}$ pesan $35,2 \%$ y $20,1 \%$

12 Conformado por actividades que presta el Estado: primero, administración pública y defensa; seguridad social de afiliación obligatoria y educación de no mercado. En segundo lugar, está la educación de mercado, que se construye a partir del número de alumnos matriculados en los municipios — preescolar, básica, media y superior- multiplicado por el valor estimado de la matrícula, entre otras.

13 Es el trabajador autónomo que desarrolla su actividad profesional de forma habitual a cambio de dinero sin un contrato laboral que le vincule formalmente a alguna empresa. Esto permite vislumbrar la precariedad laboral.

14 Prestan servicios personales y de protección relacionados con viajes, limpieza, catering, cuidado personal, protección contra incendios y actos ilícitos, o demuestran y venden productos al por mayor o al por menor en tiendas y establecimientos similares, así como en puestos ambulantes y en los mercados. 
respectivamente y no requerirían un número importante de empleados con altos niveles de escolaridad, como son los profesionales egresados de la UTP, que, en caso de ser contratados, tampoco recibirían salarios acordes con sus expectativas.

Tabla 5

Ocupados por posición ocupacional, según grupo principal de ocupación y rama de actividad, 1977

\begin{tabular}{|c|c|c|c|c|c|c|c|c|c|}
\hline $\begin{array}{l}\text { Rama de la } \\
\text { actividad } \\
\text { económica }\end{array}$ & $\begin{array}{l}\text { Trabajadores } \\
\text { de los } \\
\text { servicios }\end{array}$ & $\begin{array}{l}\text { Comerciantes } \\
\text { y vendedores }\end{array}$ & $\begin{array}{c}\text { Personal } \\
\text { administrativo }\end{array}$ & $\begin{array}{l}\text { Profesionales } \\
\text { y técnicos }\end{array}$ & $\begin{array}{c}\text { Directores y } \\
\text { funcionarios } \\
\text { públicos } \\
\text { superiores }\end{array}$ & $\begin{array}{l}\text { Trabajadores } \\
\text { agrícolas y } \\
\text { forestales }\end{array}$ & $\begin{array}{c}\text { No } \\
\text { informa }\end{array}$ & $\begin{array}{l}\text { Total por } \\
\text { rama } \\
\text { económica }\end{array}$ & $\%$ \\
\hline Agropecuaria & 381 & 0 & 1867 & 1187 & 254 & 20217 & 0 & 22318 & 2,11 \\
\hline $\begin{array}{l}\text { Minas y } \\
\text { canteras }\end{array}$ & 420 & 0 & 692 & 723 & 868 & 0 & 0 & 3201 & 0,30 \\
\hline $\begin{array}{l}\text { Industrias } \\
\text { manufactureras }\end{array}$ & 14552 & 35871 & 65211 & 19144 & 31817 & 622 & 0 & 435794 & 41,24 \\
\hline $\begin{array}{l}\text { Electricidad, } \\
\text { gas y agua }\end{array}$ & 209 & 667 & 4060 & 455 & 784 & 0 & 0 & 9433 & 0,89 \\
\hline Construcción & 3587 & 213 & 8996 & 9588 & 2639 & 207 & 0 & 136985 & 12,96 \\
\hline $\begin{array}{l}\text { Comercio, } \\
\text { restaurantes y } \\
\text { hotel }\end{array}$ & 65020 & 297630 & 59586 & 7379 & 4956 & 417 & 0 & 43866 & 4,15 \\
\hline $\begin{array}{l}\text { Transporte y } \\
\text { comunicación }\end{array}$ & 3365 & 627 & 25515 & 6125 & 6287 & 0 & 0 & 104581 & 9,90 \\
\hline $\begin{array}{l}\text { Estable } \\
\text { financiero }\end{array}$ & 21514 & 12060 & 58082 & 32512 & 7359 & 0 & 0 & 45359 & 4,29 \\
\hline $\begin{array}{l}\text { Serv. comun. } \\
\text { Soc. y pers. }\end{array}$ & 332818 & 6402 & 68165 & 133521 & 9846 & 3992 & 0 & 255020 & 24,13 \\
\hline No informa & 0 & 0 & 0 & 0 & 0 & 0 & 131 & 131 & 0,01 \\
\hline $\begin{array}{l}\text { Total por } \\
\text { posición } \\
\text { ocupacional }\end{array}$ & 441866 & 353470 & 292174 & 210634 & 64810 & 25455 & & 1056688 & \\
\hline$\%$ & 41,82 & 33,45 & 27,65 & 19,93 & 6,13 & 2,41 & & 0,00 & \\
\hline
\end{tabular}

Nota. Tomado de Boletín mensual de estadística DANE, 1977.

Otra cifra disponible es la de los desocupados ${ }^{15}$ según el nivel de escolaridad. En la tabla 6 puede observarse que los mayores porcentajes de desocupación son para la categoría primaria urbana, tanto a nivel nacional $-44,6 \%$ - como regional $-50,6 \%$-, mientras que, en la categoría de educación superior universitaria, el número de desocupados es del $0,9 \%$ y $1,2 \%$, respectivamente. Por supuesto, para la época la mayoría de la población aún no alcanzaba niveles de escolaridad muy altos. Todavía no estaba en auge la masificación de la educación superior, pero los índices de desempleo estaban latentes.

15 Los desocupados son personas que, por sus características, se espera que sean parte de la PEA. Sin embargo, no se han podido vincular al mercado laboral. El DANE los ha dividido en cesantes - los que ya han trabajado, pero ahora no tienen empleo- y aspirantes - los que por primera vez buscan trabajo-. 
Tabla 6

Desocupados por nivel de escolaridad en 1973

\begin{tabular}{lcccc}
\hline \multicolumn{1}{c}{ Nivel de escolaridad } & \multicolumn{2}{c}{ Nacional } & \multicolumn{2}{c}{ Región Central } \\
\hline Ninguna & 73430 & $12,5 \%$ & 15602 & $9,2 \%$ \\
Primaria urbana & 262203 & $44,6 \%$ & 85900 & $50,6 \%$ \\
Primaria rural & 81929 & $13,9 \%$ & 21898 & $12,9 \%$ \\
Bachillerato básico & 42018 & $7,1 \%$ & 4941 & $2,9 \%$ \\
Bachillerato clásico & 91690 & $15,6 \%$ & 33097 & $19,5 \%$ \\
Secundaria técnica o vocacional & 11496 & $2,0 \%$ & 871 & $0,5 \%$ \\
Otra secundaria & 2093 & $0,4 \%$ & 1222 & $0,7 \%$ \\
Normalista & 12938 & $2,2 \%$ & 3559 & $2,1 \%$ \\
Superior universitaria & 5440 & $0,9 \%$ & 2051 & $1,2 \%$ \\
Otra & 4251 & $0,7 \%$ & 483 & $0,3 \%$ \\
No informa & 255 & $0,0 \%$ & & \\
Total & $\mathbf{5 8 7 7 4 3}$ & & $\mathbf{1 6 9 6 2 4}$ & \\
\hline
\end{tabular}

Nota. Región Central incluye: Risaralda, Caldas, Quindío, Tolima y Huila. Tomado de Boletín mensual de estadística del DANE, 1976, (297 y 298).

Por otra parte, y para ilustrar el panorama laboral de los egresados de la UTP, en el contexto nacional, se buscaron cifras históricas del país respecto de las tasas de ocupación según el máximo nivel de escolaridad alcanzado. Los datos reportados por el DANE cubren el periodo comprendido entre 1985 hasta 2018, con una ausencia de información entre 1986 y 1990. En la gráfica 1 se observan varios comportamientos:

1. Todos los porcentajes de ocupación han bajado notablemente, excepto para la educación media — se denomina así a los grados $10^{\circ}$ y $11^{\circ}$ del bachillerato- Vale aclarar que antes del 2007, esta hacía parte de la secundaria. Se llamaba bachilleres a todos los estudiantes que terminaban los once grados de escolaridad. Luego se dividió el bachillerato en media y secundaria ${ }^{16}$. El nivel de educación media registró 26,7 puntos porcentuales, y en 2018 alcanzó 33,1 puntos.

2. Las personas ocupadas con primaria y secundaria - hasta grado $9^{\circ}$ del bachilleratohan sido las de mayor presencia en la distribución del empleo. Han estado incluso por encima del $40 \%$. Estos son los empleos que no requieren cualificación académica ${ }^{17}$ de alto nivel. Lo mismo sucede con la educación media. Valga decir que "la calidad de la educación media se considera el momento crucial para que los estudiantes accedan a oportunidades académicas y laborales, a través del desarrollo de competencias y el logro de los desempeños" (Corredor, 2019, p. 122).

16 Los tres niveles de educación formal en Colombia son: (a) el preescolar, que comprenderá mínimo un grado obligatorio; (b) la educación básica, con una duración de nueve grados que se desarrollará en dos ciclos: la educación básica primaria de cinco grados y la educación básica secundaria de cuatro grados; y (c) la educación media con una duración de dos grados.

17 Como ejemplo de ello se puede ver a nivel departamental, en una página de ofertas de empleo (www.computrabajo. com.co), que lo más frecuente en Risaralda es encontrar convocatorias para cargos como: mensajero, mercaderista, jardinero, asesor comercial, agente de centro de atención telefónica, auxiliar de enfermería, auxiliar de pintura, operario, conductor de camión, bachiller con 4 meses de experiencia, oficial de obra, asistente administrativo, técnico electrónico, impulsador, auxiliar de cocina, mecánico de mantenimiento. 
3. El porcentaje de los ocupados que tiene educación superior, en esta serie, siempre ha estado por debajo de los otros niveles educativos, aunque presentó un crecimiento sostenido hasta 2005 y alcanzó el 18,4\%. El descenso inició cuando se incluyeron los ocupados con posgrado o los de educación técnica y tecnológica, que son los que, probablemente, les competirán por los puestos de trabajo. Los ocupados con postgrado se registran desde 2007 y han estado en estos doce años por debajo de los 5 puntos porcentuales

\section{Figura 1}

Distribución porcentual de la población ocupada en Colombia según el último nivel educativo

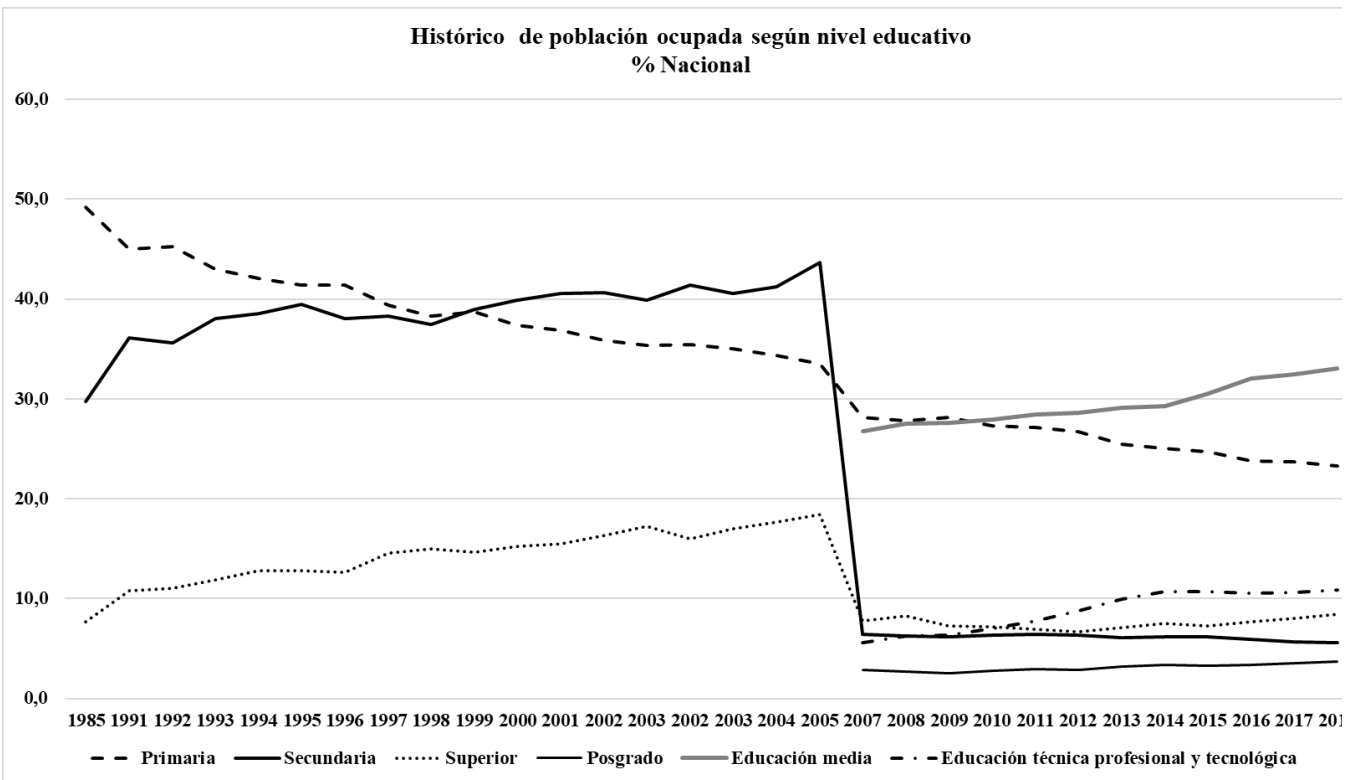

Nota: Indicadores de empleo del personal ocupado, por nivel educativo. Adaptado de (DANE, 1985 y 2018).

Sin perder de vista que el crecimiento de la educación superior responde, en gran medida, a las necesidades de desarrollo del sector económico, vale la pena mirar cómo está constituido el PIB departamental y cómo se ha reorganizado este en su interior a través del tiempo (tabla 7), porque es allí donde aportarán conocimientos los graduados de la educación superior. Los cambios más notorios en el orden de los renglones de la economía están en la agricultura, que pasó de $26,9 \%$ de participación en el PIB departamental al 6,4 \%. Esto indica que Risaralda dejó de ser un departamento agricultor, debido a la caída de los precios del café en el mercado internacional, lo que hizo poco rentable la producción del grano, así como todas las actividades productivas dependientes de él. Muchos de los empleos de este sector se reacomodaron en el sector comercial (Cepeda, 2011).

Tabla 7

Composición sectorial del PIB en Risaralda 1980, 2005 y 2018

\begin{tabular}{lccc}
\hline \multicolumn{1}{c}{ Sector económico } & $\mathbf{1 9 8 0}$ & $\mathbf{2 0 0 5}$ & $\mathbf{2 0 1 8}$ \\
\hline Agricultura, ganadería, caza, silvicultura y pesca & 26,9 & 10,6 & 6,4 \\
Explotación de minas y canteras & 0,1 & 0,8 & 0,3 \\
Industrias manufactureras & 28,4 & 15,4 & 12,2
\end{tabular}




\begin{tabular}{lccc} 
Electricidad, gas y agua & 0,4 & 3,1 & 2,5 \\
Construcción & 3,3 & 7,7 & 7,8 \\
Comercio, reparación de vehículos, transporte & 17,4 & 15,9 & 20,5 \\
Información y comunicaciones & 1,3 & 4,2 & 2,9 \\
Actividades financieras y de seguros & 7,2 & 2,8 & 3,4 \\
Actividades inmobiliarias & 5,5 & 6,1 & 6,5 \\
Actividades profesionales, científicas y técnicas & 6,0 & 8,2 & 8,9 \\
Administración pública y defensa & 4,6 & 13,2 & 16,4 \\
Actividades de entretenimiento & 0,0 & 3,6 & 3,0 \\
Impuestos & 1,6 & 8,5 & 9,2 \\
\hline \multicolumn{1}{c}{ PIB Risaralda en el contexto nacional } & 2,18 & 1,6 & 1,6 \\
\hline
\end{tabular}

Nota. Tomado de Cuentas Departamentales de Colombia del DANE, 2021.

También, en este mismo periodo de 38 años, comprendido entre 1980 y 2018, la industria manufacturera mostró una caída importante en la participación en el PIB departamental, pues pasó del 28,4\% al 12,2\%. Esto obedece en parte a que, de las 25515 empresas medias y grandes de la industria manufacturera en Colombia, solo 50 están en Risaralda y el $64 \%$ de estas últimas son de bajo nivel tecnológico (Tamayo et al., 2017), lo que puede significar poca contratación de mano de obra de alto nivel académico.

En contraste con estos dos sectores, el renglón comercial pasó de representar en el PIB de Risaralda el $1 \%$ en 1960, al 17,4 \% en 1980, hasta alcanzar el $20,5 \%$ en 2018. Este comportamiento marca un giro en la vocación económica del departamento, en respuesta a la crisis cafetera y en aprovechamiento de su ubicación estratégica, al estar en el cruce de caminos entre las grandes capitales - Bogotá, Medellín y Cali-, lo que le hace propicio atender el aprovisionamiento de un gran número de viajeros y convierte al comercio en el renglón de la economía que genera mayor empleo en el departamento (Cepeda, 2011).

\section{El escalamiento de la "generación engañada" en Risaralda}

De acuerdo con lo anterior, destacan tres renglones de la economía que tienen mayor representación dentro del PIB de Risaralda en 2018: comercio, administración pública e industria manufacturera; es allí donde se generan más puestos de trabajo. Sin embargo, particularmente en esta última en Risaralda, se registran 50 empresas y, de ellas, solo $36 \%$ son de medio o alto nivel tecnológico (Tamayo et al., 2017). Entonces, surgen algunas preguntas:

- ¿Dónde se emplearán los profesionales egresados de las IES del departamento? Estas han crecido en un $621 \%$ en el periodo de 2000 a 2018 (SNIES, 2018). Se incluyen, además de la UTP, al resto de las IES del departamento (gráfica 2).

- ¿Se viene generando un desequilibrio paulatino entre las posibilidades de vinculación al mercado del trabajo y la cantidad de jóvenes que terminan la educación superior?

- ¿Están siendo "desmentidas" las posibilidades reales de los graduados para ejercer sus profesiones? 
Según el Observatorio del Trabajo y la Seguridad Social Colombiano, "el incremento del número de trabajadores con niveles educativos superiores a los requeridos en las ocupaciones en que se desempeñan (sobre-educados) ha venido en aumento; pasando del 14,9 \% para 2006 al 19,3\% para el 2016" (Farné et al., 2017, p. 7).

\section{Figura 2}

Tendencia en el número de graduados en educación superior en el departamento de Risaralda. Periodo 2001-2018

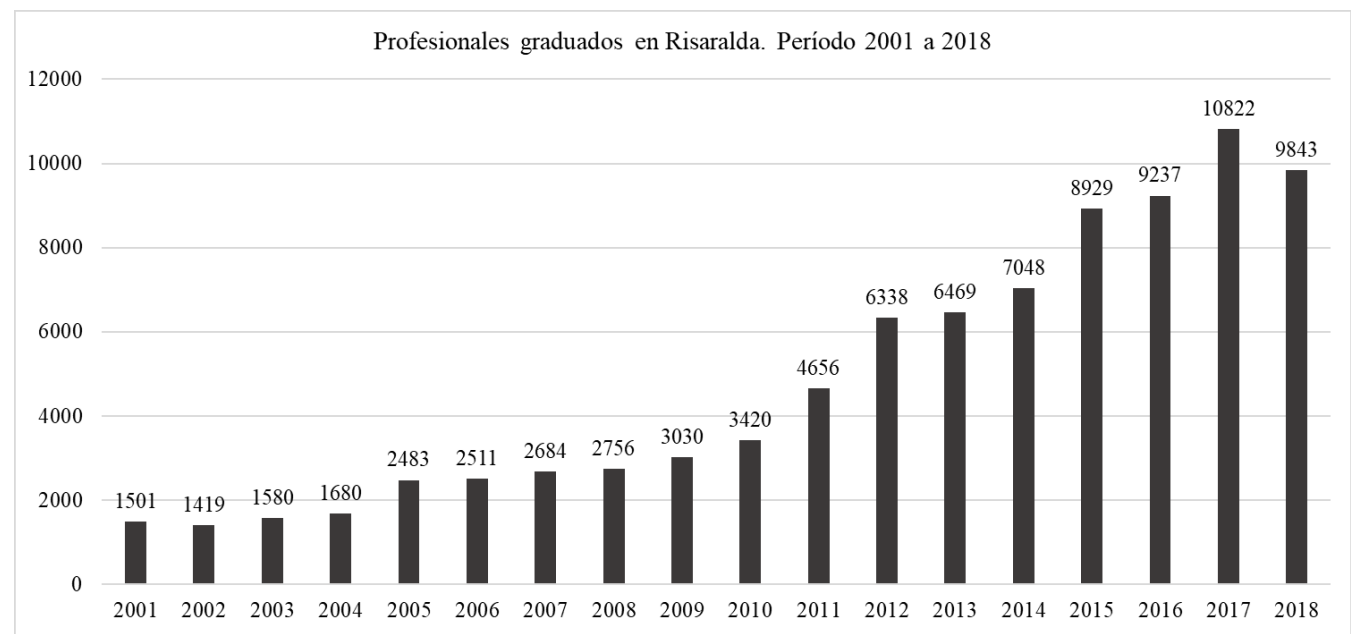

Nota. Adaptado de Bases de datos consolidadas, SNIES, 2018.

Si bien el departamento ha tenido épocas de crecimiento y desarrollo notables, no ha ido más allá del $2 \%$ de aporte al PIB nacional y su configuración interna se ha modificado de manera significativa. En este contexto histórico, económico y social, la UTP se consolidó como la principal IES en el departamento, al formar profesionales dentro de la lógica del modelo "económico-economicista" en el que están inmersos tanto la UTP como el resto de IES de Risaralda, que en 2018 eran 26. Conviene subrayar que lo esperado es que los egresados a través de sus prácticas contribuyan al desarrollo tanto propio como el de sus familias y su entorno social.

No obstante, para los profesionales que surgen de las clases populares, el empleo es el camino para mejorar condiciones de vida propias y de sus núcleos familiares. En general, en el imaginario colectivo, se reforzó "la concepción de la educación como un medio de acceso a mercados de trabajos más calificados o al circuito internacional de formación superior e intercambio" (Giovine, 2015, p. 8). Sin embargo, la "escasa empleabilidad juvenil" (Isacovich, 2019, p.19) que es factor común en Latinoamérica. En particular, el sistema laboral risaraldense viene presentando debilidad y poco dinamismo en el crecimiento durante las últimas décadas, como lo muestran las altas cifras de desempleo que en Pereira como ciudad principal del departamento, según el DANE (2020a, p. 20), ya alcanza el 24,9\% en el semestre abril-junio del 2020. Además, los empleos ofrecidos no guardan relación con las competencias y expectativas salariales de los profesionales de la región. Según Cardona (2016), "se requiere una mejora radical del sistema laboral, que considere entre otros puntos, remuneración congruente con el cargo laboral” (p. 78).

De hecho, la Asociación de Egresados —creada en 1968 en la UTP para mantener 
comunicación con los graduados y, entre otras actividades, compilar datos sobre ellosrecientemente efectuó un seguimiento al $15 \%$ de los 36092 graduados hasta diciembre de 2018. Encontró que el 60 \% estaba laborando (Buitrago et al., 2018). Entre ellos, el $81 \%$ alcanzó máximo 3 SMLV, en su primer año de vinculación. Por su parte, el MEN, en el sitio web del Observatorio Laboral del Egresado (OLE, 2019) reporta el IBC $^{18}$ estimado y la tasa ${ }^{19}$ de cotizantes para las IES del país. En particular, para los recién graduados de la UTP, el IBC es un salario promedio de \$1 710973 y la tasa de cotización para recién graduados en 2016, es de 80,7 \%. En cuanto a cifras salariales de los recién graduados, los datos de OLE y de la Asociación de Egresados coinciden en gran medida. No pasa igual con los porcentajes de ocupación. Lo que sí es un hecho es que la asociación viene ejecutando estrategias para acercar graduados y empleadores, en lo que ha denominado ruta de empleabilidad (Buitrago et al., 2018), cuyo nombre en sí es una muestra de la preocupación por contribuir en la vinculación de los graduados al mercado laboral. El trabajo informal también ha aumentado de manera notoria, principalmente en el sector terciario ${ }^{20}$, así como los contratos sin estabilidad laboral y sin garantía de prestaciones (Cardona, 2016).

Además de la baja oferta de cargos en el mercado laboral, que viene en aumento ya que "se preveía para el año 2018 un incremento de 2.7 millones de desempleados en el mundo, asegurándose que una de las principales causas de este crecimiento es el exceso de fuerza de trabajo, misma que supera a la creación de empleos" (Aguado, 2020, p. 39). También debe tenerse en cuenta que se requieren conexiones sociales relevantes para encontrar empleo, lo cual es más difícil si se es egresado de universidad pública, bajo la premisa de que quien egresa de una IES pública pertenece a las clases de origen social humilde y, por ende, no dispone de capital social suficiente para movilizar en pro de alcanzar otras posiciones más favorables en el espacio social, disponer de lazos sociales con agentes en posiciones sociales de poder favorece la consecución de empleo:

los lazos sociales que emergen de relaciones de parentesco y socialización (territorial y/o institucional) brindan recursos que pueden acumularse, trasmitirse y movilizarse a lo largo de las trayectorias de vida. Estos lazos potencian las posibilidades de transmisión de información y la incorporación de saberes y modelos de comportamiento que pueden resultar fundamentales para manejarse dentro del mercado laboral (Carrascosa y Estévez, 2020, p. 257).

En una investigación reciente sobre la desigualdad de oportunidades en Colombia, se observó como hallazgo importante que "hay un porcentaje ligeramente mayor de graduados de instituciones privadas con empleo formal, con relación a los que egresaron de instituciones públicas” (Cuenca, 2016, p. 76).

A pesar de estas cifras de desempleo y de salarios no satisfactorios, la expansión de la

18 IBC: índice base de cotización al Sistema de Seguridad Social Integral (SSSI).

19 Calculada como tasa de vinculación de cotizantes, indica la proporción de estos que se conectan al mercado laboral en cualquier región del país, que hacen aportes al SSSI, dividido entre el total de graduados del periodo t-1.

20 "El sector terciario en la economía es el que se encarga de la distribución y comercialización de los productos y servicios frente al consumidor y recoge las siguientes actividades: comercio, reparación, restaurantes y hoteles, transporte, almacenamiento, comunicaciones, establecimientos financieros, seguros, actividades inmobiliarias y servicios a las empresas, actividades de servicios sociales, comunales y personales. Según el DANE, este sector aloja el 80 \% de la fuerza laboral del país" (Martínez, 2020). 
educación superior y de la graduación ${ }^{21}$ en el país ha sido creciente. Este comportamiento incluye a Risaralda, que cuenta con un número importante de profesionales graduados. Según cifras del SNIES (2018), entre el primer semestre del 2001 y el segundo semestre de 2017, se registró el otorgamiento en Risaralda de 76563 títulos, de los cuales 62672 eran de pregrado y 13891 de posgrado. Estos títulos cubren una amplia gama de profesiones. Entre ellas se destacan, principalmente, ingenieros industriales, enfermeras, abogados y contadores públicos. En la tabla 8 se muestran las quince profesiones con más títulos otorgados en educación superior en Risaralda entre 2001 y 2017.

\section{Tabla 8}

Las 15 profesiones con más títulos otorgados en educación superior en Risaralda, entre los años 2001 y 2017.

\begin{tabular}{clc}
\hline $\begin{array}{c}\text { Puesto en el total de } \\
\text { títulos en Risaralda }\end{array}$ & \multicolumn{1}{c}{ Programa académico } & $\begin{array}{c}\text { Numero de graduados } \\
\text { 2001 a 2017 }\end{array}$ \\
\hline 1 & Ingeniería Industrial & 3094 \\
2 & Enfermería & 2662 \\
3 & Derecho & 2620 \\
4 & Contaduría Pública & 2334 \\
5 & Administración de Empresas & 2021 \\
6 & Tecnología Industrial & 1399 \\
7 & Licenciatura en Pedagogía Infantil & 1389 \\
8 & Psicología & 1369 \\
9 & Tecnología en Contabilidad y Finanzas & 1309 \\
10 & Medicina & 1261 \\
11 & Administración de Negocios Internacionales & 1239 \\
12 & Ingeniería de Sistemas y Computación & 1133 \\
13 & Ingeniería Mecánica & 1117 \\
14 & Tecnología en Gestión Administrativa & 1052 \\
15 & Ingeniería Eléctrica & 1041 \\
\hline
\end{tabular}

Nota. Adaptado de SNIES, 2018.

Los datos de la tabla 8 responden al crecimiento no planificado de la educación superior, que produce un número importante de graduados, quienes, a pesar de la deserción, se enfrentan a un entorno laboral en donde las plazas de trabajo disponibles no alcanzan a cubrir sus demandas ni en número, ni en expectativas salariales y contractuales. Ellos han asumido el desafío de cursar una carrera profesional o técnica con el fin de romper el ciclo reproductivo de pobreza y alcanzar con sus títulos profesionales otras "posiciones" dentro del espacio social que los ubique a distancia de las que ocupan en el punto de partida de sus

21 Los datos usados para producir la información se obtuvieron de las bases consolidadas disponibles en la siguiente dirección: (https://snies.mineducacion.gov.co/portal/ESTADISTICAS/Bases-consolidadas/). Se encontró que el país graduó 4800920 profesionales, en el periodo comprendido entre enero de 2001 y diciembre de 2017. De ellos, 3367950 son de pregrado. Las 10 carreras de mayor graduación fueron: Administración de Empresas -219 960_, Contaduría Pública —182 914-, Derecho —166 352-, Ingeniería Industrial — 98 466 - , Psicología — 95 640_, Ingeniería de Sistemas — 79 802_, Técnico Profesional en Servicio de Policía — 70 333-, Medicina —65 413-, Ingeniería Civil — 50 134- y Enfermería — 49 551—. 
carreras universitarias.

No obstante, en el caso de que el estudiante haga un cálculo razonado de las probables transformaciones de su realidad social como producto de la consecución de un título profesional, se va a encontrar con una sobreabundancia de títulos (tabla 8), que genera inequidad en el acceso a las oportunidades laborales. Hay que sumar a esto el hecho de que, si bien la deficiencia de capital cultural ha generado inequidad en el acceso a una educación de calidad, también es cierto que la deficiencia de capital social genera inequidad en el acceso al mundo laboral: en otras palabras, "el origen social distingue a individuos de nivel escolar idéntico" (Bourdieu, 1979, p. 103).

Sin duda, según las cifras divulgadas por los entes de gobierno y las reflexiones de varios especialistas sobre el crecimiento desbordado de cupos en la educación superior, se reconoce la validez del planteamiento de Bourdieu (1979) respecto a que se ha venido construyendo lentamente la "generación engañada"22, una generación donde la distancia entre las esperanzas subjetivas y las posibilidades objetivas (Bourdieu, 1979) se acentúa cada vez más, gracias a que hay un número mayor de graduados en las mismas disciplinas, como pudo verse en la tabla 8 , con el riesgo de convertirse en una problemática de dimensiones estructurales si no es atendida con rigor:

El aumento generalizado de la escolarización tiene como efecto el aumento de la masa del capital cultural... y el rendimiento de la acción escolar tiende continuamente a acrecentarse. Si todo lo demás... permanece igual, [se puede] producir un efecto inflacionista al hacer accesibles las titulaciones a un mayor número de personas. (Bourdieu, 1979, p. 130)

Se reitera que el riesgo del fenómeno inflacionario es latente al presentar la titulación profesional como el camino para la solución de problemas económicos, al entender que la educación, además de entregar saberes, también tiene el poder de consagrar derechos a través de la otorgación de títulos que conceden aspiraciones, que muchas veces están lejos de la realidad. "Cuando los [jóvenes] de las clases populares no estaban dentro del sistema, este no era el mismo. A causa de todo esto hay una devaluación por simple inflación y también porque cambió la 'calidad social' de los que poseen los títulos" (Bourdieu, 1984, p. 123)

Fuera del mercado propiamente escolar, la titulación vale lo que vale económica y socialmente su poseedor, siendo el rendimiento del capital escolar función del capital económico y social que puede ser dedicado a su valorización. De manera general, los cuadros [grupos ocupacionales] tienen tantas más probabilidades de acceder a las funciones de dirección en lugar de a las de producción, fabricación, mantenimiento, cuanto más alto es su origen social. (Bourdieu, 1979, p. 133)

Veintiséis años después, González (2005), en su estudio sobre el mercado laboral en el Área Metropolitana Centro Occidente $(\mathrm{AMCO})^{23}$, concluyó, en consonancia con Bourdieu,

22 El desajuste entre las aspiraciones que el sistema de enseñanza produce y las oportunidades que realmente ofrece, en una fase de inflación de las titulaciones, es un hecho estructural que afecta, en diferentes grados según la singularidad de las mismas y según el origen social, al conjunto de los miembros de una generación escolar. Las clases recientemente llegadas a la enseñanza secundaria están expuestas a esperar de ella, por el solo hecho de haber tenido acceso a la misma, lo que proporcionaba en el tiempo en que estaban prácticamente excluidas de dicha enseñanza. Estas aspiraciones que, en otros tiempos y para otro público, eran perfectamente realistas, puesto que se correspondían con unas posibilidades objetivas, son frecuentemente desmentidas, con mayor o menor rapidez, por los veredictos del mercado escolar o del mercado laboral. (Bourdieu, 1979, p. 142)

23 Esquema asociativo territorial que promueve estrategias de desarrollo regionales en conjuntos de ciudades afines. AMCO está 
que a mayor oferta laboral calificada en la región - al referirse a la de los egresados de la educación superior, con relativos niveles de experiencia, enfrentados a la poca dinámica de la demanda laboral para los puestos de trabajo acordes a su preparación, que han presionado caídas sostenidas en los salarios reales-, también es mayor el número de jóvenes desencantados por el choque contra una realidad que no era la imaginada cuando decidieron adelantar estudios superiores, con la expectativa de encontrar alternativas laborales con posibilidades de buenos ingresos económicos y no el panorama actual que ofrece el país, como es la disminución de la tasa de retorno de ingresos ${ }^{24}$.

\section{Conclusiones}

En resumen, la educación superior en Risaralda ha sido creciente, tanto en número de IES como en matrícula, pero se ha llegado a un punto de estancamiento y a una tendencia a la caída en la matrícula. Sin embargo, sigue presente un número importante de estudiantes. A pesar de la deserción, la mitad de ellos se gradúa. Entre los que se gradúan hay en común una tasa de desempleo alta y en aumento - $20 \%$ - o plazas de trabajo con tareas y salarios muy por debajo de las expectativas económicas de los profesionales. Como se evidenció a través de las cifras, en los últimos dieciséis años el departamento ha graduado más de 3000 ingenieros industriales y enfermeros; y alrededor de 2000 abogados, contadores y administradores; y números cercanos a mil en las otras profesiones. Sin embargo, la tasa de ocupación en ese sector educativo se mantiene a la baja. La única línea de tendencia de ocupación que crece ligeramente es la de la educación media - grados $10^{\circ}$ y $11^{\circ}$ del bachillerato-. Esto se corresponde con las ofertas laborales que no requieren mayor cualificación, lo que produce una especie de "desclasamiento" 25 , en el que se condena a los nuevos profesionales a una especie de "desilusión colectiva" (Bourdieu, 1979, p.142) por el incumplimiento de expectativas. También, aunque es un caso fortuito, vale la pena mencionar que, como consecuencia de la pandemia del 2020, se perdieron 61000 empleos en el departamento (DANE, 2020b), lo que complica aún más la situación en términos de pobreza monetaria y del panorama socioeconómico en general. En este momento más que nunca se requiere la ejecución de proyectos que mejoren la infraestructura económica y potencien el desarrollo industrial y agropecuario para dar impulso a la economía regional y dar posibilidades a las nuevas generaciones de egresados de la educación superior para que cumplan sus expectativas de movilidad dentro del espacio social hacia mejores posiciones, a la vez que contribuyen con el desarrollo del departamento en todas sus dimensiones.

\section{Referencias}

Aguado., L. (2020). Efecto del empleo y el desempleo en el estado de ánimo de jóvenes universitarios recién egresados. Enseñanza e Investigación en Psicología, 2(1), 38-46.

Acevedo, A., Rodríguez, D., \& Giraldo, N. (2009). Jorge Roa Martínez: memoria de una

formada por Pereira, Dosquebradas y La Virginia, al recoger el 74\% — 709.338 - de los habitantes del departamento de Risaralda.

24 Si tenemos en mente que el volumen de los puestos correspondientes puede haber variado también en el mismo intervalo, es posible considerar que una titulación tiene todas las posibilidades de haber sufrido una devaluación ya que el aumento del número de poseedores de titulaciones académicas es más rápido que el aumento del número de puestos a los que esas titulaciones conducían al principio del período considerado. (Bourdieu, 1979, p. 131)

25 Devaluación del rendimiento social de los títulos académicos. Estos se encuentran en un nivel de empleo inferior al que hubieran tenido tiempos atrás con el mismo título: "desviación respecto a la pendiente de la trayectoria colectiva” (Bourdieu, 1979, p. 111). 
visión cosmopolita. Editorial UPT.

Asamblea Departamental de Risaralda. (1968). Ordenanza 1. http://www.asamblearisaralda. gov.co/sitio/2018/01/resena-historica/

Bourdieu, P. (1979). La Distinción. Criterios y bases sociales del gusto. Taurus.

Bourdieu, P. (1984). Sociología y cultura. Editorial Grijalbo.

Bourdieu, P. (2002). Campo intelectual, campo de poder. Montressor.

Bourdieu, P., \& Wacquant, L. (2005). Una invitación a la sociología reflexiva. Siglo XXI.

Buitrago, P., Hernández, N., \& Quinceno, Y. (2018). Política y acciones de la universidad para promover la empleabilidad de los egresados. Gestión de egresados Universidad Tecnológica de Pereira. En C. Garrido, \& D. García-Pérez (Coords.), Políticas y acciones de las universidades para promover el empleo de sus egresados. Estudios de caso en Iberoamérica (pp. 68-81). Redue Alcue; Faedpyme. https://issuu.com/redue/docs/cap04_b782144a1c60ee

Carrascosa, J. \& Estévez, B. (2020). Mecanismos de acceso al empleo al comienzo de la trayectoria laboral: Desigualdades de clase, territoriales y educativas. En El análisis de clases sociales: pensando la movilidad social, la residencia, los lazos sociales, la identidad y la agencia. CLACSO: Instituto de investigaciones Gino Germani. http://biblioteca. clacso.edu.ar/Argentina/iigg-uba/20200729122828/El-Analisis-de-Clases-Sociales. pdf $\#$ page $=254$. P. 253-286

Cardona, J. et al(2016). Una mirada al mercado laboral del departamento de Risaralda mediante las variables: ocupación, desocupación, inactividad y población en edad de trabajar, periodo 2001-2014 [Tesis de Pregrado, Universidad Libre de Colombia]. Repositorio Institucional Unilibre. https://repository.unilibre.edu.co/bitstream/ handle/10901/16234/UNA\%20MIRADA\%20AL\%20MERCADO\%20LABORAL. pdf? sequence $=1$ \&isAllowed $=y$

Cepal. (2021). Acerca de la Cepal. https://www.cepal.org/es/acerca

Cepeda, L. (2011). La economía de Risaralda después del café: ¿Hacia dónde va? Reportes del emisor, 152, 1-8.

Congreso de la República. (1958, 29 de diciembre). Ley 41 de 1958. Por la cual se crea la universidad Tecnológica de Pereira. Diario Oficial n 29847. http://www.suin-juriscol. gov.co/viewDocument.asp?ruta=Leyes/1596419

Congreso de la República. (1966, 1 de diciembre). Ley 70 de1966. Por la cual se crea y organiza el Departamento de Risaralda. Diario Oficial n 32101. http://www.suin-juriscol.gov.co/ viewDocument.asp?ruta=Leyes/1620232

Correa, J. (2014). Civismo y educación en Pereira y Manizales (1925-1950): un análisis comparativo entre sus sociabilidades, visiones de ciudad y cultura cívica. UTP.

Corredor, N. (2019). Factores de la calidad educativa desde una perspectiva multidimensional: análisis en siete regiones de Colombia. Plumilla Educativa, 23(1), 121-139.

Constitución Política de Colombia [Const]. Art. 67.7 de julio de 1991 (Colombia). 
Cuenca, A. (2016). Desigualdad de oportunidades en Colombia: impacto del origen social sobre el desempeño académico y los ingresos de graduados universitarios. Estudios Pedagógicos, 42(2), 69-93.

DANE. (1938). Censo general de población. Tomo XVI. http://biblioteca.dane.gov.co/media/ libros/LD_771_1938_V_1.PDF

DANE. (1951). Censo de población de 1951. Departamento de Caldas. http://biblioteca.dane. gov.co/media/libros/LB_803_1951_V_1.PDF

DANE. (1964). Censo de población de 1964. Departamento de Caldas. http://biblioteca.dane. gov.co/media/libros/LB_803_1964.PDF

DANE. (1973). XIV Censo Nacional de Población y III de vivienda 1973. República de Colombia. Departamento de Risaralda. http://biblioteca.dane.gov.co/media/libros/ LB_807_1973.PDF

DANE. (1975). Boletín Mensual de Estadística, (287). http://biblioteca.dane.gov.co/media/ libros/BOL_287_1975-115.PDF

DANE. (1976). Boletín Mensual de Estadística, (297-298). http://biblioteca.dane.gov.co/ media/libros/BOL_297-298_1976-125.PDF

DANE. (1977). Boletín Mensual de Estadística, (312). http://biblioteca.dane.gov.co/media/ libros/BOL_312_1977-139.PDF

DANE. (1985). XV Censo Nacional de Población y IV de vivienda 1985. República de Colombia. Departamento de Risaralda. http://biblioteca.dane.gov.co/media/libros/ LB_771_1985_V_3.PDF

DANE. (1985). Indicadores de empleo del personal ocupado, por nivel educativo $(1985,2018)$. https://www.dane.gov.co/index.php/estadisticas-por-tema/mercado-laboral/empleo-ydesempleo

DANE. (1989). Cuentas departamentales de Colombia 1980-1989. http://biblioteca.dane. gov.co/media/libros/LD_9595_1980_1989_EJ_4.PDF

DANE. (2019). Resultados Censo Nacional de Población y Vivienda 2018. https://www.dane. gov.co/files/censo2018/informacion-tecnica/presentaciones-territorio/190731-CNPVpresentacion-Risaralda-Pereira.pdf

DANE (2020a). Boletín técnico. Gran Encuesta Integrada de Hogares (GEIH). Junio 2020. https://www.dane.gov.co/files/investigaciones/boletines/ech/ech/bol_empleo_jun_20. pdf

DANE. (2020b). Compendio estadístico y panorama de Risaralda, ante los impactos de la pandemia. https://tic.risaralda.gov.co/publicaciones/154259/alrededor-de-61-mil-personasperdieron-su-empleo-tras-la-pandemia-en-pereira-y-su-area-metropolitana/

DANE. (2021). Cuentas nacionales y departamentales. https://www.dane.gov.co/index.php/ estadisticas-por-tema/cuentas-nacionales/cuentas-nacionales-departamentales

Decreto 3686 de 1954 [con fuerza de ley].(1954, 22 de diciembre). Por el cual se provee el desarrollo de las Universidades colombianas. Diario Oficial $n^{\circ} 28667$. 
Escobar, N. (2018). Limitantes sociales y políticos de la industrialización por sustitución de importaciones en Colombia 1950-1980. Universidad Nacional de Colombia.

Farné, S., Nieto, A., \& Ríos, P. (2017). Mercado laboral y educación: desajuste educativo en Colombia. Universidad Externado de Colombia.

Giovine, M. (2015). La privatización de la educación y las clases dominantes del espacio social del Gran Córdoba [Ponencia]. XI Jornadas de Sociología. Facultad de Ciencias Sociales, Universidad de Buenos Aires, Buenos Aires, Argentina.

González, O. (2005). Análisis del comportamiento del mercado laboral en el área Metropolitana Centro Occidente. http://www.almamater.edu.co/sitio/Archivos/Documentos/ Documentos/00000089.pdf

Hurtado, J. (2018). Incidencia de los periodos de industrialización por sustitución de importaciones y de apertura económica en la generación de ciencia, tecnología e innovación en Colombia. Revista Nova et Vetera, 4(41). https://www.urosario.edu.co/ Revista-Nova-Et-Vetera/Omnia/Incidencia-de-los-periodos-de-Industrializacion-po/

Isacovich, P. (2020). El PROGRESAR y la apuesta por la educación superior como abordaje de las juventudes. BORDES, (15), 17-25. https://publicaciones.unpaz.edu.ar/OJS/index. php/bordes/article/view/595

Martinez, C. (2020, 31 de agosto). Sector Terciario en Colombia: Actividades Principales. Lifeder. https://www.lifeder.com/sector-terciario-colombia/.

OLE. (2019). Oferta regional. http://bi.mineducacion.gov.co:8380/eportal/web/menobservatorio-laboral/instituciones-de-educacion-superior-ies-

Rama, G. (1970). El sistema universitario en Colombia. Editorial Universidad Nacional de Colombia.

Sociedad de Mejoras Públicas de Pereira. (2021). Historia. https://www.smpereira.org/es/ conoce-la-sociedad-de-mejoras-de-pereira

SNIES. (2018). Bases de datos consolidadas (2001, 2018). https://snies.mineducacion.gov. co/portal/ESTADISTICAS/Bases-consolidadas/

Tamayo, J., Ardila, J., \& Ocampo, C. (2017). Análisis de las empresas medianas y grandes de la industria manufacturera de la región del eje cafetero colombiano. Revista Espacios, 38(19), 1-19. https://www.revistaespacios.com/a17v38n19/a17v38n19p08.pdf

UTP. (2001). Información estadística 1961-1964. https://www2.utp.edu.co/planeacion/ documentos/boletin_1961-1964.pdf

Zuluaga, V. (2013). Historia extensa de Pereira. Editorial UTP. 